\title{
CHEMOPREVENTIVE EFFECT OF OLIVE OIL IN EXPERIMENTALLY INDUCED HAMSTER BUCCAL POUCH EPITHELIAL DYSPLASIA (RANDOMIZED CONTROL TRIAL)
}

\author{
Mohamed Abdelrahman Mohamed", Kamal Abd EL-Rahman Kamal** \\ and Abd Elnasser Abd El Mola Esmail ${ }^{* * *}$
}

\begin{abstract}
The progression of tumor depends on the level of Bcl-2 which protects the cells from undergoing apoptosis, and its level should be more than the level of the death inducer. So, our Aim: To investigate chemopreventive effect of olive oil in epithelial alteration which induced in hamster buccal pouch.
\end{abstract}

Methods: Epithelial dysplasia will occur by the application of DMBA on alternative days for 8 weeks on hamster buccal pouch. Hematoxylin and Eosin (H\&E) and Streptavidin-biotin immunoperoxidase staining techniques were used to detect the expression of Bcl-2 in three groups (G1 negative control or normal group, G2 DMBA group and G3 olive oil chemoprevention group). Evaluation of the immunostaining was done by using computer image analyzer system.

Results: H \& E stained histological section in G1 with normal mucosa of HBP intact, and continuous epithelium of thin keratinized stratified squamous epithelium while in G2 there were incidence of severe epithelial dysplasia with hyperkeratosis \& G3 reduced dysplasia incidence and severity as compared to G2. Bcl-2 immunohistochemical expression in G1 in basal \& suprabasal layers while in $\mathrm{G} 2$ \& G3 located in all epithelial layers with statistically highly significant difference $(\mathrm{P}<0.01)$ in means values of Bcl-2 expression among $(\mathrm{G} 2>\mathrm{G} 1, \mathrm{G} 3)$ and $(\mathrm{G} 3>\mathrm{G} 1)$.

Conclusion: Olive oil plays an important role in reduction and prevention of transformation of normal oral epithelium into epithelial dysplasia. The liquid form of olive oil may be considered as a new line of chemoprevention in oral dysplasia and the Bcl-2 expression evidenced this reduction of abnormality in epithelial dysplasia.

KEYWORDS: Oral epithelial dysplasia, chemopreventive effect, olive oil, bcl2, induced hamster dysplasia.

\footnotetext{
* Associate Professor, Oral pathogy, Faculty of Dentistry, AlAzhar University, Cairo, Boys

** Lecturer of Oral and Dental Pathology, Department of Oral and Dental Pathology, Faculty of Dental Medicine (Cairo - Boys) Al-Azhar University, Egypt

*** Associate Professor, Oral histology, Faculty of Dentistry, AlAzhar University, Cairo, Boys
} 


\section{INTRODUCTION}

In gauge medical nomenclature, abnormality of development means dysplasia, but the histological features of dysplasia characterized by epithelial cellular and structural changes ${ }^{(1)}$. The most common sign of premalignant lesions is dysplastic changes which is considered as the main histopathological features of epithelial premalignant lesions (2). Abnormal activity in normal epithelium is considered as a histopathological feature of dysplasia. The term dysplasia was inserted by Reagon in 1958 in relation to cells exfoliated from the uterine cervix ${ }^{(3)}$. The existence of epithelial dysplasia play an important role in predicting malignant progression than the clinical characteristics ${ }^{(4,5)}$. The progression of cancer in the epithelium of the upper aerodigestive tract is believed to be associated with the presence of dysplastic areas. The cellular atypia and wastage of normal ripeness and stratification are the common characteristic histopathological dysplastic features of a stratified squamous epithelium ${ }^{(6)}$. The transformation to canceris believed to be associated with the presence of dysplastic areas in the oral mucosa. Interestingly, the rate of mild epithelial dysplasia transformation to malignant form is similar to that of severe dysplasia ${ }^{(7)}$. The suppression of these lesions to transform into malignant tumors is considered as the essential goal of management of pre-malignant lesions. Until now there is no solidarity about what the best treatment of oral dysplasia to inhibit the progression of malignancies. Cancer is considered as one of the most horrible diseases and act as the 2nd major cause of death all over the world with circa 14 million new cases and 8.2 million cancer-related deaths in $2011^{\left({ }^{(8)}\right.}$. Diet play an important role in cancer and computation for about $30-35 \%$ of cancer jeopardy so cancer could be effectively inhibited by modifying dietary patterns ${ }^{(9)}$. One of the fundamentals of human diet are Mediterranean dietary fats (MDF) and the essential ingredient of dietary fat is olive oil. Since ancient time different cultures believe that, the olive fruit has been looked upon as a signal of hope, peace and sacredness ${ }^{(10)}$. Olive oil (Oleaeuropaea, Oleaceae), virgin olive oil and extra virgin olive oil is anessentialingredient of the MDF; it is a mix of fatty acids such as oleic and linoleic acid, secoiridoids (oleuropein and oleocanthal), simple phenols (tyrosol andhydroxytyrosol), lignans (pinoresinol), flavonoids (apigenin), hydrocarbons (squalene), triterpenes (maslinic acid), and phytosterols ( $\beta$-sitosterol). The extraction techniqueapplied to obtain oil from the olives play an important role in chemical structure of olive oil. By applying high pressure, the olive oil is separated from the fruit core after olives crushed. Throwing, post-pression or re-pression, with or without the use of hot water is considered as additional processes. But in these additional methods the olive oilshows a sturdycolor, feeble flavour, and a higher concentration of free fatty acids ${ }^{(11,12)}$. Olive oil is including two main fractions, major fraction and minor fraction. By weight, the major fraction represents more than $98 \%$ of oil and mostly consists of mono- and poly-unsaturated fatty acids. Fatty acid component of other seed oils iscompletely various from that ofolive oil. The concentration of oleic acid in olive oil ranges from $56 \%-84 \%$ of total fatty acids which considered as one of mono-unsaturated fatty acids (MUFAs) that present in high amount in olive oil ${ }^{(13)}$. Also, olive oil contains very small amounts of saturated fatty acids such as stearic, palmitic acids (8\%-14\%) and omega- 3 fatty acids such as $\alpha$-linolenic acids ${ }^{(13,14)}$.

The living cells contain genes that normally play a role in the regulation of cell proliferation and function if these cells exposed to mutational alteration lead to uncontrolled cell growth thus leading to cancer. The genes implicated in tumorigenesis include the regulatory gens which regulate the cell proliferation (either promoting or inhibiting), apoptotic genes that controlled cell death and finally genes which responsible for repair of damaged DNA. Reliant on how they influence every process, these genes are named as 
tumor suppressor genes (growth inhibitory), protooncogenes (growth promoting), or anti-apoptotic genes (inhibits apoptosis) ${ }^{(15)}$. One of anti-apoptotic protein is B-cell lymphoma/leukemia-2 (Bcl-2) that interacts with and is regulated by $\mathrm{p} 53$.The progression of tumor depends on the level of Bcl-2 which protects the cells from undergoing apoptosis, and its level should be more than the level of the death inducer, bax ${ }^{(16)}$. The present study was carried out to investigate chemopreventive effect of olive oil in epithelial alteration (Epithelial dysplasia) \& carcinogenesis which induced in hamster buccal pouch.

\section{MATERIAL AND METHODS}

15 golden Syrian male hamsters five weeks old, by weighting 80-120 gms were used as experimental animals. They were obtained from animal house, Faculty of Pharmacy, Cairo, Boys Al Azhar University. The experiment was carried out according to the ethical \& research committee protocol of the Faculty of Pharmacy, Cairo, Boys Al Azhar University. The procedure were conducted in accordance with the committee for purpose of control and supervision on experiment on animals (CPCSEA Guide lines) $)^{(17)}$ The animals were randomly divided into three groups (G1, G2 and G3): G1 (negative control or normal group): 5 animals were left untreated. G2 (DMBA: 2,4-Dimethoxybenzaldehyde group): 5 hamsters were included in this group, the right hamster buccal pouch (HBP) of these hamsters were painted with 0.5 DMBA in paraffin using a number 4 camel hair brush three times a week for 8 weeks. G3 (olive oil chemoprevention group) 5 hamsters were included in this group were received dose of olive oil $(1 \mathrm{ml} / 100 \mathrm{~g} \text { body weight })^{(18)}$ given by the oral route using a specific vehicle one week before, as well as during the application of DMBA on alternative days for 8 weeks. After termination of the experiment, all animals were euthanized, and the buccal mucosa was excised and prepared for both histological examination by $\mathrm{H} \& \mathrm{E}$ and immunohistochemical examination by Bcl-2. The immunostaining procedures by $\mathrm{H} \& \mathrm{E}$ and immunohistochemically by $\mathrm{Bcl}-2$ the immunostaining procedure was performed according to the manufacturer instructions ${ }^{(19)}$. The immunostained sections were examined using light microscope to assess the prevalence of positive cases and the localization of immmunostaining within the tissues. In addition, image analysis computer system was used to assess area percentage of positive cells of the immunostaining. This was done in the Oral and Dental Pathology Department - Faculty of Dental Medicine - Boys- Cairo - AlAzhar University. The degree of positive staining for each antibody was evaluated by a well-established semi-quantitative scoring on a scale range from negative to strong positive staining as follow: Strong staining (more than $50 \%$ stained), moderate staining (between 25 and $50 \%$ stained), weak staining (between 5 and $25 \%$ stained), and negative (less than $5 \%$ stained) ${ }^{(20)}$. Statistical presentation and analysis of the present study was conducted, using the mean, standard deviation, ANOVA and Tukey's test by SPSS V204. Significant level: Non-Significant $>0.05$ Significant $<0.05^{*}$ High Significant $<0.01 *$.

\section{RESULTS}

Group1(Normalgroup): Normal clinical features of HBP mucosae were pink in color with smooth surface and no observable abnormalities (Fig.1.A). $\mathrm{H} \& \mathrm{E}$ stained histological section of G1 showed, normal mucosa of HBP intact, and continuous epithelium composed of thin keratinized stratified squamous epithelium. Subepithelial connective tissue (C.T), muscular layer and areolar layer were seen (Fig.1.B). Bcl-2 immunohistochemical stained section of G1 showed weak (6.710\%) positive expression which limited to basal and suprabasal layers (Fig.1.C). Group 2 (DMBA group): Clinical picture of HBP showed tumor mass and ulcerative lesion in mucosa (Fig.2.A). H\&E 
stained histological section of G2 showed 100\% of cases incidence of severe epithelial dysplasia with hyperkeratosis, acanthosis, elongation and broadrete pegs with criteria of dysplasia, included loss of adhesion, hyperchromatism and abnormal mitosis with intact basement membrane (Fig.2.B). Immunohistochemical stained (Bcl-2) section of G2 showed strongly positive staining $(53.45 \%)$ which located in all epithelial layers (Fig.2.C). Group 3 (chemoprevention group): Clinical picture of HBP showed white discoloration without ulceration and bleeding (Fig.3.A). H\&E stained histological section of G3 showed reduced epithelial dysplasia incidence and severity as compared to G2, Three exhibited mild epithelial dysplasia while two specimen appeared apparently normal almost the same as G1 (Fig.3.B). Immunohistochemical stained (Bcl-2) section of G3 showed mild (weak) positive staining $(53.45 \%)$ which located in all epithelial layers (Fig.3.C).

\section{Statistical analysis}

Results were revealed that, in regard to expression of Bcl-2, G2 had recorded the highest mean area percentage $(53.45 \%)$, while G1 had recorded the lowest mean area percentage $(6.710 \%)$ and there was high significant difference between G1,G2, and G3 where P value was $<0.01$, also there was high significant difference between the following groups (G1 and G2), ( (G2 and G3) and (G1 and $\mathrm{G} 3)$, respectively where $\mathrm{P}$ value was $(<0.01)$ (Table 1, Chart 1).

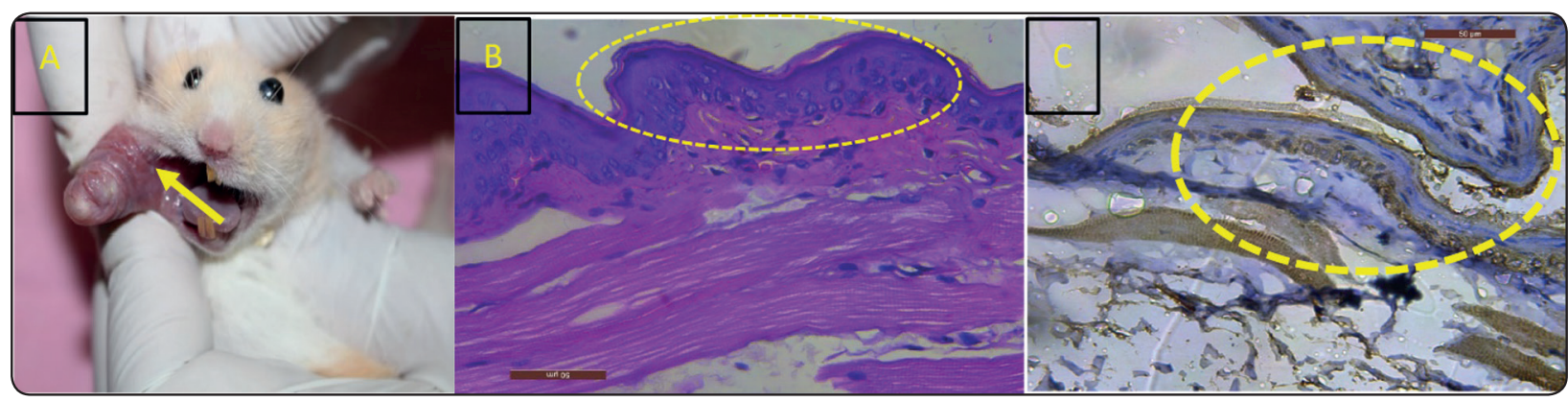

Fig. 1 A) Clinical features of G1 the yellow arrows focused on HBP mucosae were pink in color with smooth surface. B) H \& E histological section of G1 the yellow dotted area focused on, intact, and continuous epithelium of HBP mucosae (X 200). C) Bcl-2 immunohistochemical stained section of G1 the yellow dotted area focused on weak staining which limited in basal and suprabasal layers (X 200).

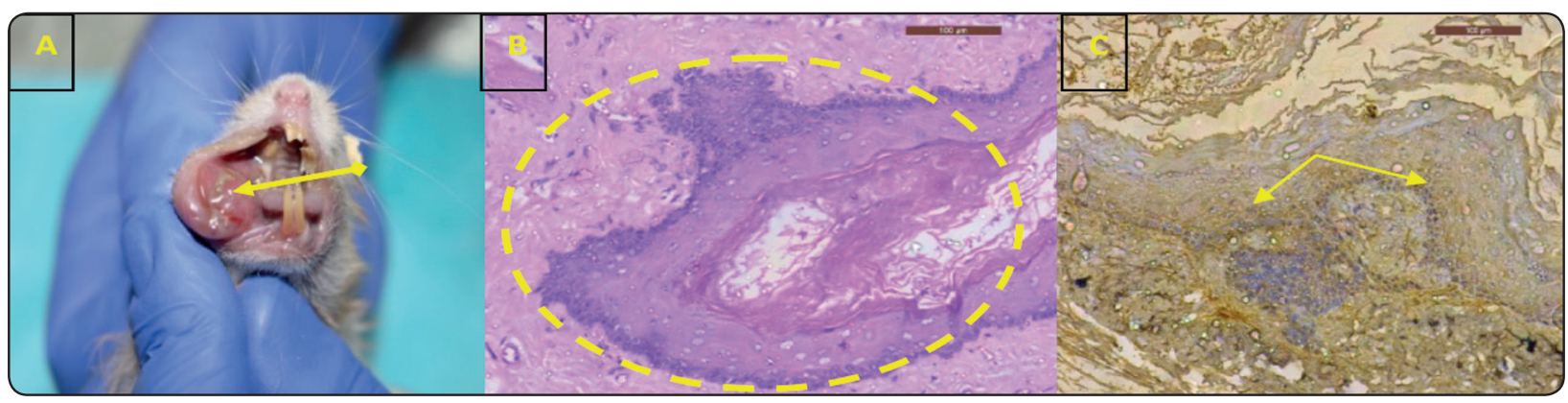

Fig. 2 A) Clinical picture of $\mathrm{G} 2$ the yellow arrows focused on HBP with tumor mass and ulcerative lesion in mucosa. B) H\&E stained histological section of G2 the yellow dotted area focused on severe epithelial dysplasia with hyperkeratosis, acanthosis, elongation and broad rete pegs with criteria of epithelial dysplasia(X 200). C) Immunohistochemical stained (Bcl-2) section of G2 the yellow arrows focused on strongly positive staining in all epithelial layers (arrow) (X 200). 


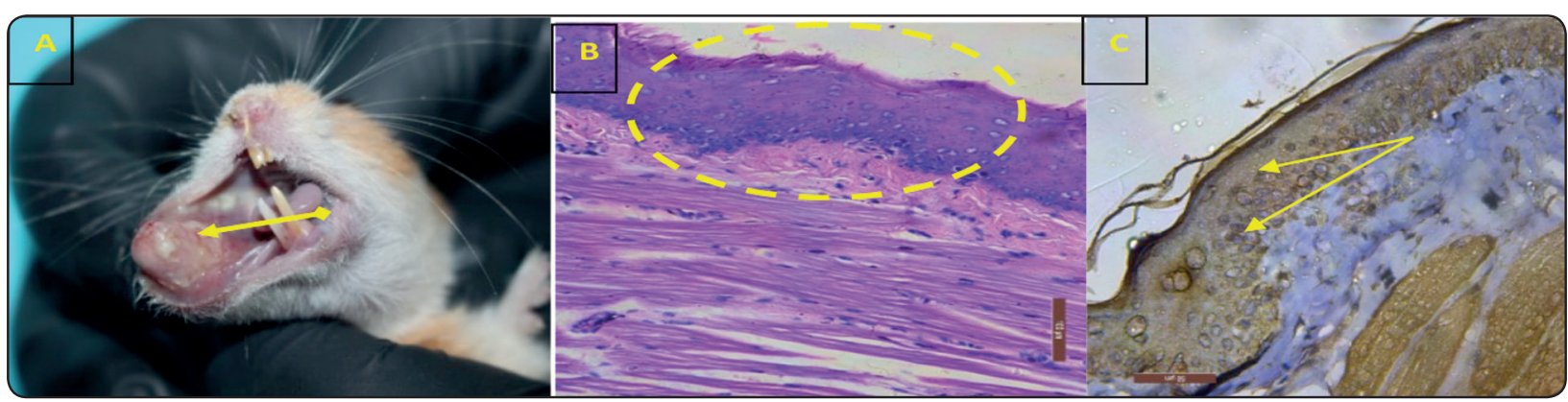

Fig. 3 A) Clinical picture of G3 the yellow arrows focused on HBP with white discoloration without ulceration and bleeding. B) H\&E stained histological section of G3 the yellow dotted area focused on mild epithelial dysplasia (X 200). C) Immunohistochemical stained (Bcl-2) section of $\mathrm{G} 3$ the yellow arrows focused on mild (weak) positive staining which located in all epithelial layers (arrow) (X 200).

TABLE (1) Statistical analysis between normal (G 1), DMBA group (G 2) and olive oil + DMBA group (G 3) by ANOVA and Tukey's test.

\begin{tabular}{|c|c|c|c|c|c|}
\hline & $\begin{array}{c}\text { (I) } \\
\text { Grouping }\end{array}$ & $\begin{array}{c}(\mathbf{J}) \\
\text { Grouping }\end{array}$ & $\begin{array}{c}\text { Mean } \\
\text { Difference } \\
(\mathbf{I}-\mathbf{J})\end{array}$ & $\begin{array}{l}\text { Std. } \\
\text { Error }\end{array}$ & Sig. \\
\hline \multirow{6}{*}{ 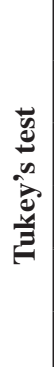 } & \multirow{2}{*}{ G1 } & G2 & $-46.74000^{*}$ & .20471 & .000 \\
\hline & & G3 & $-8.58000^{*}$ & .20471 & .000 \\
\hline & \multirow{2}{*}{ G2 } & G1 & $46.74000^{*}$ & .20471 & .000 \\
\hline & & G3 & $38.16000^{*}$ & .20471 & .000 \\
\hline & \multirow{2}{*}{ G3 } & G1 & $8.58000^{*}$ & .20471 & .000 \\
\hline & & G2 & $-38.16000^{*}$ & .20471 & .000 \\
\hline \multirow{3}{*}{ z } & \multicolumn{2}{|c|}{ G1 } & 6.710 & 0.3624 & \multirow{3}{*}{$<0.01 *$} \\
\hline & \multicolumn{2}{|c|}{ G2 } & 53.45 & 0.3808 & \\
\hline & \multicolumn{2}{|c|}{ G3 } & 15.29 & 0.1949 & \\
\hline
\end{tabular}

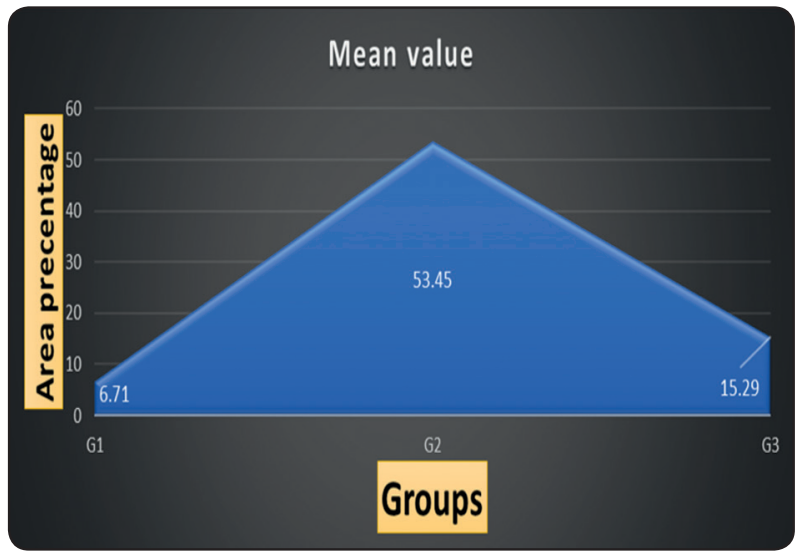

Chart 1: Mean value of area percentage of normal group $(\mathrm{G} 1)$, DMBA group $(\mathrm{G} 2)$ and olive oil + DMBA group $(\mathrm{G} 3)$

\section{DISCUSSION}

Oral epithelial dysplasia is considered as abnormal, atypical proliferation encountered fundamentally in the epithelium. Epithelial atypia, dyskeratosis and epithelial dysplasia were the terms used synonymously ${ }^{(21)}$. The term "dysplasia" is generally regard as the perception of a disordered development. In a stratified squamous epithelium, the normal stratification and maturity affected by architectural disorder ${ }^{(22)}$. The epithelial dysplasia of the oral mucosa is believed to be accompanied bythe cancer progression associated. The sever epithelial dysplasia may be progressed to malignancy in an individual lesion. However, non-dysplastic lesions may also show malignant growth ${ }^{23,24)}$. Mainly clinical features of epithelial dysplasia appeared as a red, white or mixed lesion. There is an association between the histological severity of the lesion and the risk of malignant transformation, so the histological grading plays an important role in the transformation ${ }^{(25)}$. In the present study, all over the world olive oil is considered as one of the most common components of Mediterranean dietary fat so we used it to investigate its ability as a malignant transformation preventive and anti-carcinogenic agent. Gross observation of G3 model revealed white discoloration without ulceration and bleeding but G2 tumor mass and ulcerative lesion in mucosa. Also, in G3 reduced epithelial dysplasia incidence, three specimen with mild dysplastic while two specimen 
apparently normal almost the same as G1, also there was high significant difference between (G3 and $\mathrm{G} 2)$, where $P$ value was $(\mathrm{p} \leq 0.01)$ regarding to $\mathrm{Bcl} 2$ expression. The progression of tumor depends on the level of Bcl-2 which protects the cells from undergoing apoptosis ${ }^{(16)}$, our results reported the antagonist of chemopreventive substance (olive oil) with Bcl-2 expression.

This result similar to the observations of Borzì $M$ et al (26), described thatthe olive oil contains substances which supports the chemotherapeutic possibility against colorectal cancer (CRC), these substances acting on different ways, such as oxidative damage, inflammation and even epigenetic modulation. Previous study supported that nutritional compounds play an important role in cancer initiation and development ${ }^{(27)}$, Psaltopoulo et al ${ }^{(28)}$ revealed that, consumption of olive oil was accompanied with reduction of cancer development, managed to investigate the possible chemoprotective and anticancer effect of extra virgin olive oil (EVOO) in Mediterranean diet and this result is similar to our result in present study. Dalvi et al (29) who reported that the consumption of EVOO has been accompanied with diminished hazard of several cancers particularly those of upper digestive and respiratory cancers. Fezai et al ${ }^{\left({ }^{(3)}\right)}$ reported that a significant and promising inhibition of the tumor by EVOO activity in a colon cancer xenograft model assessment. It has been demonstrated that treatment of human colon adenocarcinoma cells with phenolic compounds resulted in the inhibition of all colon carcinogenetic processes such as initiation, promotion, and metastasis, triggering cell death by apoptosis ${ }^{(31,32)}$.

The present study suggested the chemopreventive effect of olive oil against oral epithelial dysplasia. The antitumor activity seems to be related to the modulation in colonic mucosa of arachidonic acid metabolism and prostaglandin E2 synthesis, exerted by $\mathrm{n} 9$ and $\mathrm{n} 3$ fatty acids (oleic acid and eicosapentaenoic acid respectively) present in olive oil ${ }^{(33)}$. The phenolic compounds in olive oil, besides to anti-inflammatory properties, showed also immunomodulatory effects. The immunomodulatory properties could reduce chronic inflammation in inflammatory bowel diseases (IBD) and also in other immune-mediated pathologies, such as multiple sclerosis, psoriasis, rheumatoid arthritis, systemic lupus erythematosus and inflammatory bowel diseases ${ }^{(34)}$. The primarily involved cells in the autoimmune and inflammatory responses are $\mathrm{T}$ lymphocytes and antigen presenting cells (APCs), which are B cells monocyte/macrophages and dendritic cells ${ }^{(35)}$. Raised levels of inflammatory cytokines (i.e., TNF-alpha, IL-8, IL-10, IL-6, IL17) and activation of innate adaptive immune cells are involved in the pathogenesis and evolution of Inflammatory bowel diseases (IBD). On this basis, cytokine pathways modulating drugs could be used in IBD, although side effects and symptoms recurrence are common ${ }^{(36)}$. Dietary olive oil phenols seem to change clinico-pathological history in IBD, due to their anti-inflammatory properties ${ }^{(37)}$.

\section{RECOMMENDATIONS}

After termination of the current study we recommended that the researchers must apply more research work on chemopreventive substances (Olive oil alone or combined with another chemopreventive substance) as a line of prevention of the oral cancer.

\section{CONCLUSION}

Olive oil plays an important role in reduction and prevention of transformation of normal epithelium into epithelial dysplasia. The liquid form of olive oil may be considered as a new line of chemoprevention in oral dysplasia and the Bcl-2 expression evidenced this reduction of abnormality in epithelial dysplasia. 


\section{REFERENCES}

1. Zerdoner D. The Ljubljana classification-its application to grading oral epithelial hyperplasia. J Craniomaxillofac Surg. 2003; 31:75-79.

2. Bouquot JE, Speight PM, Farthing PM. Epithelial dysplasia of the oral mucosa diagnostic problems and prognostic features. Current Diagnostic Pathology. 2006; 12:11-21.

3. Rastogi V, Puri N, Mishra S, Arora S, Kaur G, Yadav L. An insight to epithelial dysplasia. Int J Head Neck Surg. $2013 ; 4: 74-82$.

4. Panjwani S, Sadiq S. p53 expression in benign, dysplastic and malignant oral squamous epithelial lesions. Pak J Med Sci. 2008; 24:130-5.

5. Lumerman H, Freedman P, Kerpel S. Oral epithelial dysplasia and the development of invasive squamous cell carcinoma. Oral Surg Oral Med Oral Pathol Oral RadiolEndod. 1995; 79:321-9.

6. Pindborg JJ, Reichart P, Smith CJ, Van der Waal I. World Health Organization: histological typing of cancer and precancer of the oral mucosa. Berlin: Springer-Verlag, 1997.

7. Holmstrup P, Vedtofte P, Reibel J, Stoltze K. Long-term treatment outcome of oral premalignant lesions. Oral Oncol. 2006; 42:461-74.

8. Kontou N, Psaltopoulou T, Panagiotakos D, Dimopoulos MA, Linos A. The Mediterranean diet in cancer prevention: a review. J Med Food. 2011; 14: 1065-78.

9. Cardeno A, Sanchez-Hidalgo M, Alarcon-de-laLastra C. An up-date of olive oil phenols in inflammation and cancer: molecular mechanisms and clinical implications. Curr Med Chem. 2013; 20: 4758-76.

10. Rafehi H, Ververis K, Karagiannis TC. Mechanisms of action of phenolic compounds in olive. J Diet Suppl. 2012; 9: 96-109.

11. Fernandez AG, Adams MR, Fernandez-Diez MJ. Table Olives: Production and Processing; Springer: New York, USA, 1997; ISBN 978-0-412-71810-6.

12. Gorzynik-Debicka M, Przychodzen P, Cappello F, KubanJankowska A, MarinoGammazza A, Knap N, Wozniak M, et al. Potential Health Benefits of Olive Oil and Plant Polyphenols. Int. J. Mol. Sci. 2018, 19, 686-99.

13. Gómez Caravaca AM, Carrasco Pancorbo A, CañabateDíaz B, Segura Carretero A, Fernández Gutiérrez
A. Electrophoretic identification and quantitation of compounds in the polyphenolic fraction of extra-virgin olive oil. Electrophoresis. 2005; 26: 3538-51.

14. Rodriguez-Morato J, Boronat A, Kotronoulas A, Pujadas M, Pastor A, Olesti E et al. Metabolic disposition and biological significance of simple phenols of dietary origin: hydroxytyrosol and tyrosol. Drug Metab Rev. 2016; 48: 218-36.

15. Rahmani A, Alzohairy M, Babiker AY, Rizvi MA, Elkarimahmad HG. Clinicopathological significance of PTEN and bcl 2 expressions in oral squamous cell carcinoma. Int J ClinExpPathol. 2012; 5: 965-71.

16. Korsmeyer SI. Regulatorsofcelldeath.TrendsGenet. 1995; 11:101-5.

17. Pereira S, Tettamanii M. Ahimsa and Alternatives- the concept of the 4th R. The CPCSEA in India. Altex. 2005;22(1/05):3.

18. Antunes LMG, Takahashi CS. Olive oil protects against chromosomal aberrations induced by doxorubicin in wistar rat bone marrow cells.genetics and molecular biology. 1999; 2: 225-27.

19. Rahman F, Bhargava A, Tippu SR, Kalra M, Bhargava N, Kaur I et al. Analysis of the immunoexpression of $\mathrm{Ki}-67$ and Bcl-2 in the pericoronal tissues of impacted teeth, dentigerous cysts and gingiva using software image analysis. Dent Res J (Isfahan). 2013 Jan-Feb; 10(1): 31-37.

20. Negi A, Puri A, Gupta R, Nangia R, Sachdeva A, Mittal M. Comparison of immunohistochemical expression of antiapoptotic protein survivin in normal oral mucosa, oral leukoplakia, and oral squamous cell carcinoma. Patholog Res Int 2015; 2015:1-6.

21. Sharma N, Hosmani JV, Tiwari V. Epithelial dysplasia: different grading system and its applications. J Int Oral Health. 2010; 1: 1-16.

22. Kujan O, Richard JO, Khattab A, Stephen A. Evaluation of a new binary system of grading oral epithelial dysplasia for prediction of malignant transformation. Oral Oncol. 2006; 42:987-93.

23. Gale N, Plich BZ, Sidransky D, Westra W, Califano J. Tumours of the hypopharynx, larynx and trachea (Epithelial precursor lesions).In: Barnes L, Eveson JW, Reichart P and Sidransky D, eds.World Health Organization Classification of Tumours Pathology \& Genetics Head and Neck Tumours International Agency for Research on Cancer (IARC) (Lyon: IARC Press),2005;. 140-43. 
24. Warnakulasuriya S, Reibel J, Bouquot J, Dabelsteen E. Oral epithelial dysplasia classification systems: predictive value, utility, weaknesses and scope for improvement. J Oral Pathol Med. 2008; 37: 127-33.

25. Lee JJ, Hong WK, Hittelman WN, Mao L, Lotan R, Shin $\mathrm{DM}$, et al. Predicting cancer development in oral leukoplakia: ten years of translational research. Clin.Cancer Res. 2000; 6:1702-10.

26. Borzì AM, Biondi A, Basile F, Luca S, Vicari ESD, Vacante M. Olive oil effects on colorectal cancer. Nutrients $2019 ; 11,32 ; 1-16$.

27. Comba A, Maestri DM, Berra MA, Garcia CP, Das UN, Eynard AR et al. Effect of $\omega-3$ and $\omega-9$ fatty acid rich oils on lipoxygenases and cyclooxygenases enzymes and on the growth of a mammary adenocarcinoma model. Lipids Health Dis. 2010; 9:112: 1-11.

28. Psaltopoulou T, Kosti RI, Haidopoulos D, Dimopoulos M, Panagiotakos DB. Olive oil intake is inversely related to cancer prevalence: a systematic review and a meta-analysis of 13800 patients and 23340 controls in 19 observational studies. Lipids in Health and Disease. 2011; 10:127:1-16.

29. Dalvi TB, Canchola AJ, Horn-Ross PL. Dietary patterns, Mediterranean diet, and endometrial cancer risk. Cancer Causes Control. 2007; 18: 957- 66.

30. Fezai M, Senovilla L, Jemaà M, Ben-Attia M. Analgesic, anti-inflammatory and anticancer activities of extra virgin olive oil.J Lipids. 2013; 1-7.
31. Gill CI, Boyd A, McDermott E, McCann M, Servili M, Selvaggini $\mathrm{R}$ et al. Potential anti-cancer effects of virgin olive oil phenols on colorectal carcinogenesismodels in vitro. Int J Cancer.2005; 20; 117:1-7.

32. Hashim YZ1, Rowland IR, McGlynn H, Servili M, Selvaggini R, Taticchi A. Inhibitory effects of olive oil phenolics on invasion in humancolon adenocarcinoma cells in vitro, Int J Cancer. 2008; 122:495-500.

33. Bartolí R, Fernández-Bañares F, Navarro E, Castellà E, Mañé J, Alvarez M, Pastor C, Cabre E, Gassull MA. Effect of olive oil on early and late events of colon carcinogenesis in rats: Modulation of arachidonic acid metabolism and local prostaglandin E(2) synthesis. Gut 2000; 46: 191-99.

34. Santangelo C, Vari R, Scazzocchio B, De Sanctis P, Giovannini C, D’Archivio M, et al. Anti-inflammatory Activity of Extra Virgin Olive Oil Polyphenols: Which Role in the Prevention andTreatment of Immune-Mediated Inflammatory Diseases? Endocr. Metab. Immune Disord. Drug Targets 2018;18, 36-50.

35. Guo, B. IL-10 Modulates Th17 Pathogenicity during Autoimmune Diseases. J. Clin. Cell Immunol.2016; 7: 400- 16.

36. Abraham C, Dulai PS, Vermeire S, Sandborn WJ. Lessons Learned from Trials Targeting Cytokine Pathways in Patients with Inflammatory Bowel Diseases. Gastroenterology $2017 ; 152,374-88$.

37. Farzaei, M.H.; Rahimi, R.; Abdollahi, M. The role of dietary polyphenols in the management of inflammatory bowel disease. Curr. Pharm. Biotechnol. 2015; 16, 196-210. 